\title{
Spectroscopy of the Ionizing Sources of UC HII Regions
}

\author{
Margaret M. Hanson ${ }^{1}$ \\ Dept. of Physics, Univ. of Cincinnati, Cincinnati, OH 45221 USA
}

\begin{abstract}
The utility of near-infrared, spectroscopic studies of central ionizing sources of UC HII regions is presented, in conjunction with a recently available, sophisticated atmospheric code, to constrain the physical conditions and environment of very massive stars at extremely early stages of evolution.
\end{abstract}

\section{Introduction}

The greatest stumbling block for developing a theory for the formation of massive stars is the lack of direct observations of massive stars at very early evolutionary stages. This is due to the very fast contraction time for massive stellar cores. While radio and millimeter observations can directly observe conditions of the collapsing material, only shorter wavelength observations, which become possible later in the formation of massive stars, are capable of directly detecting the star.

Unfortunately, by the time a massive star can be observed in the optical, critical signatures present in the spectrum and environment of the star, which yield clues to the formation process, have disappeared. We must observe massive stars before their strong ionizing winds dissipate their accretion disks and outflows, before their stellar atmospheres erase chemical and physical evidence tracing their formation mechanism (due to mixing and spin-down from masslosing winds), and before the central cluster disperses from destruction of the local molecular cloud. To investigate massive star formation, young massive star systems must be studied while they are still deeply embedded in an environment of gas and dust. The most important observations for this purpose are taken at near-infrared wavelengths because $\mathrm{OB}$ stars show few photospheric features at wavelengths greater than $2.2 \mu \mathrm{m}$. The $\mathrm{H}$ - and $\mathrm{K}$-band spectral regions, from $1.5-2.2 \mu \mathrm{m}$, are thus the most effective wavelengths which can reasonably penetrate the shrouded environment of the ultra-compact HII (UC HII) region and allow us to derive the characteristics of massive stars at their earliest evolutionary phase.

The strategy of our work is to obtain high-quality near-infrared spectra of extremely young and heavily shrouded OB-stars, and to explore quantitative analyses capable of deriving the physical conditions of these very young massive stars while they are still heavily buried in their birth clouds.

\footnotetext{
${ }^{1}$ Visiting Astronomer, Very Large Telescope, European Southern Observatory.
} 

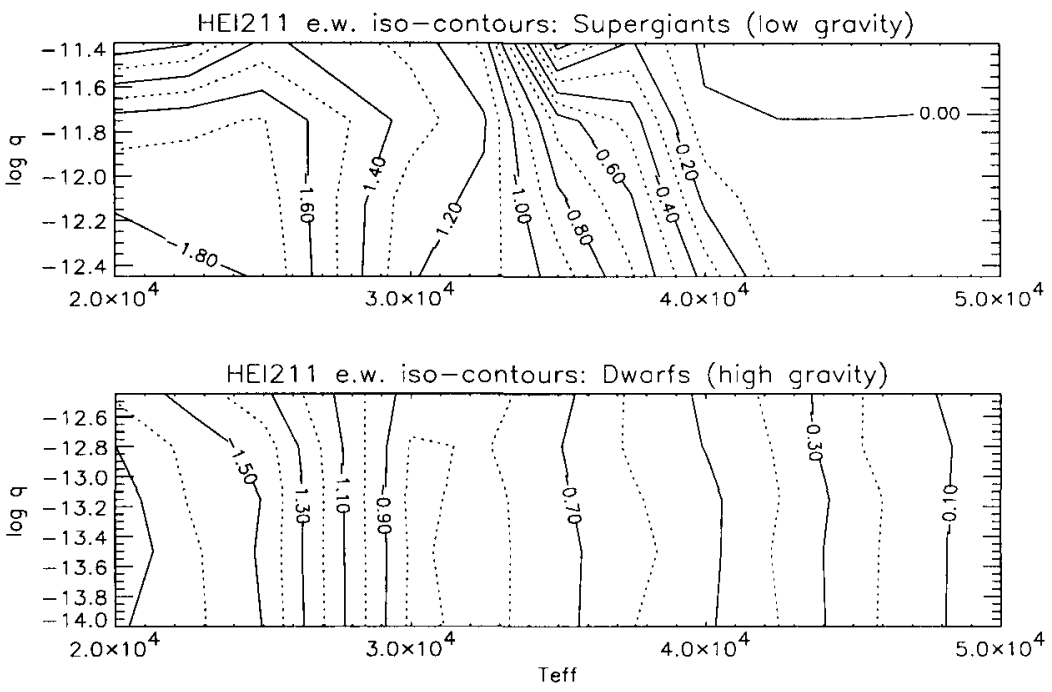

Figure 1. The vertical iso-contours of equivalent width for highgravity (dwarf) stars indicate that the HeI $2.112 \mu \mathrm{m}$ line is an excellent temperature indicator (from Puls 2000, in prep). For supergiants with extreme winds ( $\log q$ represents a parameterization related to mass loss, see Puls et al. 1996), we will need to first constrain the wind strength in conjunction with measurements of $\operatorname{Br} \gamma$.

\section{Near-Infrared Quantitative Spectroscopic Analyses}

A critical component of our program is the concurrent development of sophisticated model atmospheres which can be used with our near-infrared spectra. Puls (2000, in prep) has recently extended the non-LTE, Unified Model Atmosphere code of Santolaya-Rey, Puls \& Herrero (1997) to include near-infrared line formation and the influence of metallic background continua. These models are spherically extended, and include the important effects of winds. They yield the entire sub- and supersonic atmospheric structure. Presently this code is among the only one in existence which is able to reproduce exactly hydrostatic, non-LTE atmospheres in the limit of very small mass-loss rates, and able to model atmospheres with intermediate to very strong winds. The first step in the analysis is to constrain the stellar and wind conditions by measuring the equivalent widths (E.W.) of several strategic lines, and comparing them with model grids of E.W. One such grid is shown in Figure 1. There are a total of seven Helium and Hydrogen lines in the $\mathrm{H}-$ and $\mathrm{K}$-band spectral regions. Our initial results suggest that these lines are enough for us to consistently constrain the stellar and wind parameters for $\mathrm{O}$ and early-B stars, $T_{\mathrm{eff}}, v \sin i, L, \log g$, and mass, and to search for binaries and possible disk or in-fall signatures in very young, massive stars, relying on near-infrared spectroscopy alone. 


\section{Observations}

To locate the rare and very young massive stars needed for our study, Lex Kaper, Fernando Comeron and myself have undertaken a near-infrared imaging survey using the NTT/SOFIA. Our survey comes from a selection of radio and mid-infrared (IRAS) identified deeply embedded UC HII regions in the southern hemisphere. Nearly every field we observed showed a star forming region of interest. These images serve as guides for locating the central ionizing sources, which we believe will be among the youngest of the most massive stars accessible to near-infrared study and analyses. Furthermore, our images give us a glimpse at the rarely measured, yet highly important, stellar density of very young clusters. Based on the success of that imaging survey, our group was granted time on the VLT using the near-infrared spectrometer, ISAAC, to obtain high resolution, high signal-to-noise spectra of the central ionizing stars. These stars are among the youngest, massive stars ever to be directly studied.

\section{Final Remarks}

The very fast contraction time of massive stars sets up an immense radiation field early in the stars formation, and may reverse the in-fall of additional mass once the star reaches $10-20 \mathrm{M}_{\odot}$. This "radiation pressure problem" is so severe, it has led some theorists to suggest that stars more massive than this cannot form from accretion alone, but instead form from collisions of intermediatemass stars, which first formed through accretion (Bonnell, Bate \& Zinnecker 1998). Our goal is to make observations capable of differentiating between these two current theories for massive star formation: accretion and coalescence. The earlier the phase of evolution we can directly observe, the better hope we have of detecting the short-lived characteristics unique to each star formation scenario.

Near-infrared imaging studies have identified numerous potential young OB stars, but only a few stars have been observed spectroscopically, and these studies have relied on a comparative analysis (Hanson et al. 1997, Watson \& Hanson 1997). However, it should soon be possible to derive more accurate stellar characteristics as sophisticated atmospheric models extend their analysis to the near-infrared regime. These characteristics make up a vital boundary condition constraining theories on massive star formation.

Acknowledgments. I am grateful to Joachim Puls, Lex Kaper and Fernando Comeron, without whom this work would not have been possible.

\section{References}

Bonnell, I. A., Bate, M.R., \& Zinnecker, H., 1998, MNRAS, 298, 93

Hanson, M.M., Howarth, I.D., Conti, P.S., 1997, ApJ, 489, 698

Puls, J., Kudritzki, R.P, Herrero, A., et al., 1996, A\&A 305, 171

Santolaya-Rey, A.E., Puls, J., Herrero, A., 1997, A\&A, 323, 488

Watson, A.M., \& Hanson, M.M., 1997, ApJ, 490, L165 